IFIC/04-45, FTUV/04-0803

\title{
Pentaquark from QCD sum rules: consequences of the diquark approach
}

\author{
M. Eidemüller* a \\ a Departament de Física Teòrica, IFIC, Universitat de València - CSIC, \\ Apt. Correus 22085, E-46071 València, Spain
}

In this work we investigate the consequences of the Jaffe and Wilczek diquark model in the framework of QCD sum rules. An analysis of the $\Theta^{+}(1540)$ as $(u d)^{2} \bar{s}$ state shows that the mass of the pentaquark is compatible with the experimentally measured value. The mass difference between the $\Theta^{+}$and the pentaquark with the quantum numbers of the nucleon amounts to $70 \mathrm{MeV}$ and is consistent with the interpretation of the $N(1440)$ as a pentaquark.

Recently, several experiments [1] have observed a new baryon resonance $\Theta^{+}(1540)$ with positive strangeness. Therefore it requires an $\bar{s}$ and has a minimal quark content of five quarks. The $\Theta$ has the third component of isospin zero and the absence of isospin partners suggests strongly that the $\Theta$ is an isosinglet what we also assume in this work. A puzzling characteristics of the $\Theta$ is its narrow width below $15 \mathrm{MeV}$. A suggestive way to explain the small width is by the assumption of diquark clustering. The formation of diquarks presents an important concept and has direct phenomenological impact [2]. In this work we investigate the diquark model by Jaffe and Wilczek $[3,4]$ in the framework of QCD sum rules where the $\Theta$ is described as bound state of an $\bar{s}$ with two highly correlated $(u d)$-diquarks. The basis of the sum rules was laid in [5] and their extension to baryons was developed in [6]. The assumptions of the model are incorporated by an appropriate current. Since the sum rules are directly based on QCD and keep the analytic dependence on the input parameters, they can help to differentiate between the models and to test their features. The relevance of the diquark picture within the context of the sum rules was shown in [7]. Several sum rule investigations for the pentaquark already exist [8-14] which, however, are based on

\footnotetext{
${ }^{*}$ Talk given at the High-Energy Physics International Conference in Quantum Chromodynamics (QCD 2004), Montpellier, July 2004
}

different models or currents. The diquark models for the pentaquark have also been investigated within other approaches [15].

In the model by Jaffe and Wilczek the $(u d)$ diquarks have zero spin and are in a $\overline{3}_{c}$ and $\overline{3}_{f}$ representation of colour and flavour. In order to combine with the antiquark into a colour singlet, the two diquarks must combine into a colour 3 . The diquark-diquark wavefunction is antisymmetric and has angular momentum one. This combines with the spin of the $\bar{s}$ to total angular momentum $1 / 2$ and results in positive parity. In [3] it was suggested to interpret the Roper resonance $N(1440)$ as $(u d)^{2} \bar{d}$ pentaquark state and we will study this resonance at the end of our analysis.

The basic object in our sum rule analysis is the two-point correlation function

$\Pi(p)=i \int d^{4} x e^{i p x}\langle 0|T\{\eta(x) \bar{\eta}(0)\}| 0\rangle$,

where $\eta(x)$ represents the interpolating field of the pentaquark under investigation.

The diquarks have a particularly strong attraction in the flavour antisymmetric $J^{P}=0^{+}$channel. Thus the current contains two diquarks of the form

$\mathcal{Q}^{c}(x)=\epsilon^{a b c} Q_{a b}(x)=\epsilon^{a b c}\left[u_{a}^{T} C \gamma_{5} d_{b}\right](x)$.

$C$ denotes the charge conjugation matrix. The two diquarks must be in a $p$-wave to satisfy Bose statistics. Therefore the current contains 
a derivative to generate one unit of angular momentum. The diquarks couple to a $3_{c}$ in colour to form the current

$$
\begin{aligned}
\eta(x)= & \left(\epsilon^{a b d} \delta^{c e}-\epsilon^{a b c} \delta^{d e}\right)\left[Q_{a b}\left(D^{\mu} Q_{c d}\right)\right. \\
& \left.-\left(D^{\mu} Q_{a b}\right) Q_{c d}\right] \gamma_{5} \gamma_{\mu} C \bar{s}_{e}^{T},
\end{aligned}
$$

where the covariant derivative for the $\overline{3}_{c}$ is given by $D^{\mu}=\partial^{\mu}-i g \lambda_{l}^{\dagger} A^{\mu l}$ [4]. The parity is positive. This current has a different structure than the currents in other pentaquark sum rules [9-14] which contain no derivative to produce the angular momentum between the diquarks. Inserting the current and neglecting higher orders in the strong coupling constant the correlator is given by

$$
\begin{aligned}
\Pi(x) & =\langle 0|T\{\eta(x) \bar{\eta}(0)\}| 0\rangle \\
& =\left[\gamma_{5} \gamma^{\mu} C S_{e^{\prime} e}^{(s) T}(-x) C \gamma^{\nu} \gamma_{5}\right] T_{\mu \nu}^{e e^{\prime}}(x),
\end{aligned}
$$

where $S^{(s)}(x)$ represents the strange quark propagator. The quark propagator has been evaluated in the presence of quark and gluon condensates in $[11,16,17]$, where the explicit expressions can be found. Using the following Lorentz decomposition for $T_{\mu \nu}^{e e^{\prime}}=\delta^{e e^{\prime}} T_{\mu \nu} / 3$,

$T_{\mu \nu}=g_{\mu \nu} f_{1}\left(x^{2}\right)+x_{\mu} x_{\nu} f_{2}\left(x^{2}\right)$,

the functions $f_{1}\left(x^{2}\right)$ and $f_{2}\left(x^{2}\right)$ can be determined up to operators of dimension 6 [8]. In momentum space the correlator can be parametrised as

$\Pi(p)=\not p \Pi^{(p)}\left(p^{2}\right)+\Pi^{(1)}\left(p^{2}\right)$.

To obtain the phenomenological side we insert intermediate baryon states with the corresponding quantum numbers. Since no experimental information on higher pentaquark states is available we make the assumption of quark-hadron duality and approximate the higher states by the perturbative spectral density above a threshold $s_{0}$. In fact, the uncertainty on $s_{0}$ will be one of the dominant errors in the sum rule analysis.

In order to suppress the higher dimensional condensates and to reduce the influence of the higher resonances we employ a Borel transformation with Borel parameter $M$. As in $[10,11]$ we now concentrate on the chirality even part $\Pi^{(p)}$

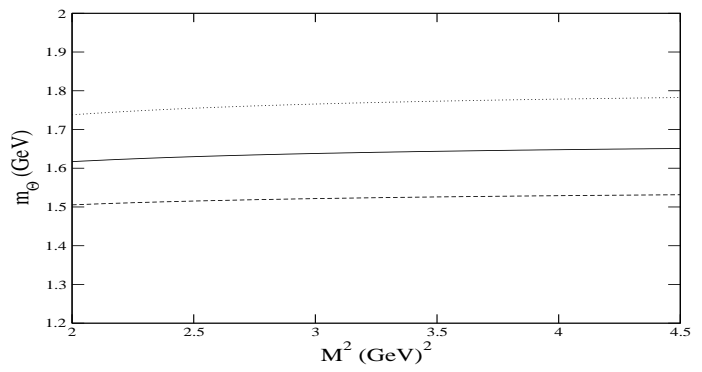

Figure 1. $m_{\Theta}$ as a function of the Borel parameter $M^{2}$ for different $s_{0}=3.5 \mathrm{GeV}^{2}$ (solid), $s_{0}=4.1 \mathrm{GeV}^{2}$ (dotted) and $s_{0}=3.0 \mathrm{GeV}^{2}$ (dashed).

in eq. (6) which contains the leading order term from the operator product expansion. Transferring the continuum contribution to the theoretical side and taking a logarithmic derivative with respect to $-1 / M^{2}$, one obtains the sum rule for the mass of the pentaquark,

$$
m_{\Theta}^{2}=\frac{\sum_{k=0}^{k=3} a_{6-k} \Gamma(8-k)\left(M^{2}\right)^{8-k} E_{7-k}}{\sum_{k=0}^{k=3} a_{6-k} \Gamma(7-k)\left(M^{2}\right)^{7-k} E_{6-k}},
$$

where $E_{\alpha}=1-\Gamma\left(\alpha+1, s_{0} / M^{2}\right) / \Gamma(\alpha+1)$.

A basic input for the sum rule analysis is the Borel parameter $M$. We employ a sum rule window of $2.5 \mathrm{GeV}^{2}<M^{2}<4.0 \mathrm{GeV}^{2}$ where the operator product expansion converges well and the phenomenological continuum is not too large. For the continuum threshold we use a central value of $s_{0}=(1.54+0.35 \mathrm{GeV})^{2}$. Thus the continuum starts $350 \mathrm{MeV}$ above the measured pentaquark mass. This difference should roughly correspond to one radial excitation [10] and represents a typical value for sum rule analyses with light quarks as degrees of freedom [5]. Fig. 1 shows the mass as a function of the Borel parameter $M^{2}$. The sum rule has a good stability with respect to $M$. As central value for the pen- 
taquark mass we obtain $m_{\Theta}=1.64 \mathrm{GeV}$. To estimate the error on $m_{\Theta}$ we vary $s_{0}$ between $3.0 \mathrm{GeV}^{2}<s_{0}<4.1 \mathrm{GeV}^{2}$. In fig. [1 we have also plotted the change of $m_{\Theta}$ with the continuum threshold from which we obtain an error of $\Delta m_{\Theta} \approx 125 \mathrm{MeV}$. To estimate the dependence of the sum rules on the OPE we successively remove the different orders. The inclusion of the higher condensates lowers the mass. The fourdimensional condensates lower the leading order result by about $50 \mathrm{MeV}$ and the condensates of dimension 6 by another $50 \mathrm{MeV}$. We assume that a reasonable error estimate from the OPE would be $\Delta m_{\Theta} \approx 75 \mathrm{MeV}$. Furthermore, contributions to the error also arise from the other input parameters which we vary in the ranges presented above. As it turns out, their influence on the value of $m_{\Theta}$ is small compared to the errors from the continuum threshold and the convergence of the OPE. Adding the errors quadratically our final result reads

$m_{\Theta}=1.64 \pm 0.15 \mathrm{GeV}$.

In [3] Jaffe and Wilczek suggested to interpret the Roper resonance as $(u d)^{2} \bar{d}$ pentaquark state. One can then perform a similar analysis for the $N(1440)$ as has been done for the $\Theta$ by substituting the $\bar{s}$ antiquark by a $\bar{d}$ antiquark. As central value for the continuum threshold we choose, as in the $\Theta^{+}$case, a value of $350 \mathrm{MeV}$ above the ground state mass. For the error range we use $2.7 \mathrm{GeV}^{2}<s_{0 N}<3.8 \mathrm{GeV}^{2}$. Performing a sum rule analysis for the $N$ with the above given parameters, we obtain a mass of $m_{N}=$ $1.57 \pm 0.15 \mathrm{GeV}$. Similar as it has been done in [11], in fig. 2 we plot the mass difference $m_{\Theta}-m_{N}$ for different values of the continuum thresholds. The mass splitting between the pentaquark states comes out to be about $70 \mathrm{MeV}$. The error represented in fig. 2 is based on the assumption that the continuum thresholds have the same offset for both pentaquark states. Phenomenologically, these values can be different and one should add to the error a part of the uncertainty from $s_{0}$ given in fig. 1] Thus the error can easily amount to $50 \mathrm{MeV}$. Though the mass difference is consistent with the interpretation of the $N(1440)$ as a pentaquark, the uncertainty re-

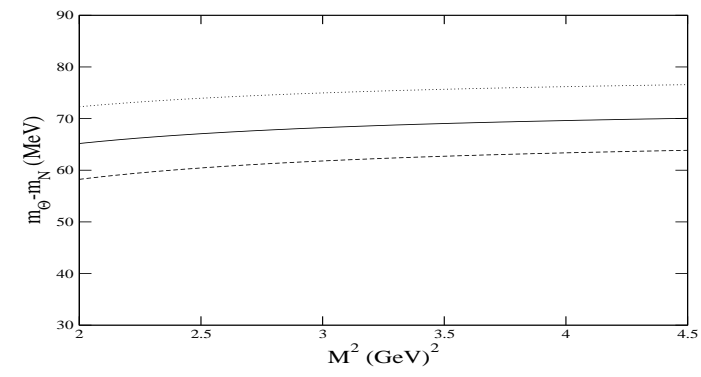

Figure 2. Mass difference $m_{\Theta}-m_{N}$ for different values of the continuum threshold, the solid, dashed and dotted lines are for $s_{0 \Theta}=3.5 \mathrm{GeV}^{2}$ and $s_{0 N}=3.2 \mathrm{GeV}^{2}, s_{0 \Theta}=4.1 \mathrm{GeV}^{2}$ and $s_{0 N}=3.8 \mathrm{GeV}^{2}$ and $s_{0 \Theta}=3.0 \mathrm{GeV}^{2}$ and $s_{0 N}=2.7 \mathrm{GeV}^{2}$, respectively.

mains large and a reduction of the error would be essential to clarify the situation.

To summarise, we have performed a QCD analysis based on the approach by Jaffe and Wilczek. We obtain a sum rule that is stable over the Borel parameter $M$ and reproduces the mass of the pentaquark within errors. We have also performed an analysis for the pentaquark with the quantum numbers of the nucleon and have shown that the interpretation of the Roper resonance $N(1440)$ as $(u d)^{2} \bar{d}$ pentaquark state is consistent with the sum rules. It is important to note that the sum rules are directly based on QCD and thus, apart from the structure of the current, do not contain further model assumptions. It would be interesting to see if lattice calculations could confirm these findings. First lattice calculations exist [18] which, however, are based on different interpolating currents and whose results are not yet conclusive. A comparison and discussion can be found in [19]. Hopefully, future experimental and theoretical investigations could further clarify and explore the pentaquark states and the consequences of the different models. 


\section{Acknowledgments}

I would like to thank S. Narison for the invitation to this pleasant and interesting conference. I thank the European Union for financial support under contract no. HPMF-CT-2001-01128. This work has been supported in part by EURIDICE, EC contract no. HPRN-CT-2002-00311 and by MCYT (Spain) under grant FPA2001-3031.

\section{REFERENCES}

1. T. Nakano et al. [LEPS Collaboration], Phys. Rev. Lett. 91 (2003) 012002. V. V. Barmin et al. [DIANA Collaboration], Phys. Atom. Nucl. 66 (2003) 1715 [Yad. Fiz. 66 (2003) 1763]. S. Stepanyan et al. [CLAS Collaboration], Phys. Rev. Lett. 91 (2003) 252001. J. Barth et al. [SAPHIR Collaboration], Phys. Lett. B 572 (2003) 127. V. Kubarovsky et al. [CLAS Collaboration], Phys. Rev. Lett. 92 (2004) 032001 (Erratum-ibid. 92 (2004) 049902). A. Airapetian et al. [HERMES Collaboration], Phys. Lett. B 585 (2004) 213. A. E. Asratyan, A. G. Dolgolenko and M. A. Kubantsev, arXiv:hep-ex/0309042 A. Aleev et al. [SVD Collaboration], arXiv:hep-ex/0401024 [ZEUS Collaboration], arXiv:hep-ex/0403051 M. AbdelBary et al. [COSY-TOF Collaboration], arXiv:hep-ex/0403011 [WA89 Collaboration], arXiv:hep-ex/0405042

2. B. Stech, Phys. Rev. D 36 (1987) 975, M. Neubert and B. Stech, Phys. Lett. B 231 (1989) 477, M. Anselmino, E. Predazzi, S. Ekelin, S. Fredriksson and D. B. Lichtenberg, Rev. Mod. Phys. 65 (1993) 1199.

3. R. L. Jaffe and F. Wilczek, Phys. Rev. Lett. 91 (2003) 232003.

4. R. Jaffe and F. Wilczek, arXiv:hep-ph/0401034

5. M. A. Shifman, A. I. Vainshtein and V. I. Zakharov, Nucl. Phys. B 147 (1979) 385, Nucl. Phys. B 147 (1979) 448, L. J. Reinders, H. Rubinstein and S. Yazaki, Phys. Rept. 127 (1985) 1, S. Narison, World Sci. Lect. Notes Phys. 26 (1989) 1.

6. B. L. Ioffe, Nucl. Phys. B 188 (1981) 317
[Erratum-ibid. B 191 (1981) 591], Z. Phys. C 18 (1983) 67, Y. Chung, H. G. Dosch, M. Kremer and D. Schall, Phys. Lett. B 102 (1981) 175, Nucl. Phys. B 197 (1982) 55, H. G. Dosch, M. Jamin and S. Narison, Phys. Lett. B 220 (1989) 251.

7. H. G. Dosch, M. Jamin and B. Stech, Z. Phys. C 42 (1989) 167, M. Jamin and M. Neubert, Phys. Lett. B 238 (1990) 387.

8. M. Eidemüller, arXiv:hep-ph/0404126 to be published in Phys. Lett. B.

9. J. Sugiyama, T. Doi and M. Oka, Phys. Lett. B 581 (2004) 167.

10. S. L. Zhu, Phys. Rev. Lett. 91 (2003) 232002.

11. R. D. Matheus, F. S. Navarra, M. Nielsen, R. Rodrigues da Silva and S. H. Lee, Phys. Lett. B 578 (2004) 323.

12. R. D. Matheus, F. S. Navarra, M. Nielsen and R. R. da Silva, arXiv:hep-ph/0406246

13. P. Z. Huang, W. Z. Deng, X. L. Chen and S. L. Zhu, Phys. Rev. D 69 (2004) 074004.

14. Y. Kondo, O. Morimatsu and T. Nishikawa, arXiv:hep-ph/0404285.

15. K. Cheung, arXiv:hep-ph/0308176 I. M. Narodetskii, Y. A. Simonov, M. A. Trusov and A. I. Veselov, Phys. Lett. B $\mathbf{5 7 8}$ (2004) 318. E. Shuryak and I. Zahed, arXiv:hep-ph/0310270 F. E. Close, arXiv:hep-ph/0311087 J. J. Dudek, arXiv:hep-ph/0403235 N. I. Kochelev, H. J. Lee and V. Vento, arXiv:hep-ph/0404065 D. Melikhov, S. Simula and B. Stech, Phys. Lett. B 594 (2004) 265.

16. V. A. Novikov, M. A. Shifman, A. I. Vainshtein and V. I. Zakharov, Fortsch. Phys. 32 (1985) 585.

17. K. C. Yang, W. Y. P. Hwang, E. M. Henley and L. S. Kisslinger, Phys. Rev. D 47 (1993) 3001.

18. F. Csikor, Z. Fodor, S. D. Katz and T. G. Kovacs, JHEP 0311 (2003) 070. S. Sasaki, arXiv:hep-lat/0310014, T. W. Chiu and T. H. Hsieh, arXiv:hep-ph/0403020 arXiv:hep-ph/0404007. N. Mathur et al., arXiv:hep-ph/0406196

19. F. Csikor, Z. Fodor, S. D. Katz and T. G. Kovacs, arXiv:hep-lat/0407033 